\title{
Subsurface ice on Mars with rough topography
}

\author{
Oded Aharonson ${ }^{1}$ and Norbert Schorghofer ${ }^{2}$ \\ Received 10 November 2005; revised 10 July 2006; accepted 12 July 2006; published 22 November 2006.
}

[1] High-latitude ground ice on Mars discovered by the Gamma Ray Spectrometer suite is thought to be thermally stable owing to the presence of vapor in the Martian atmosphere. However, local slopes can alter surface and subsurface temperatures substantially, and hence allow ground ice to persist at locations where it would otherwise be unstable. Global statistics of the topography of Mars are computed, processed, and extrapolated to derive a description of surface roughness on spatial scales to which ground ice should be sensitive. This slope distribution is convolved with a new thermal model for the dependence of subsurface ice on slope, to produce a prediction of the global ice distribution that includes the effect of topographic roughness. In the highest latitudes, slopes reduce the amount of buried ice, while in lower latitudes the ice fraction increases, widening the geographic boundary of the ice table. At the high latitudes, where ice is stable beneath horizontal ground, the estimated reduction of ice is small compared to the existing ice volume. Areas in the midlatitudes with high surface roughness that have previously been predicted to be ice free are predicted to contain quantities of ice that may be detectable at present and accessible in the future. Slopes cause ground ice to be stable to latitudes of about 25 degrees in both hemispheres, including, for example, areas within the northern Olympus Mons aureole deposits, Hecates Tholus, and Hellas basin. Ice is unstable at equatorial latitudes, even when accounting for surface slopes.

Citation: Aharonson, O., and N. Schorghofer (2006), Subsurface ice on Mars with rough topography, J. Geophys. Res., 111, E11007, doi:10.1029/2005JE002636.

\section{Introduction}

[2] Spacecraft observations of a vast reservoir of subsurface ice on Mars [Boynton et al., 2002; Feldman et al., 2002; Mitrofanov et al., 2002; Feldman et al., 2004] provide unique insight into the current state and dynamics of the Martian water cycle. Data analysis and models suggest the ground ice is the result of recent precipitation and glaciation, and of ongoing atmosphere-regolith diffusive exchange.

[3] Models have been previously constructed to predict the global spatial distribution and burial depth of ground ice based on vapor equilibrium between the atmosphere and the subsurface. The degree of sophistication of these models progressed from conceptual descriptions [Leighton and Murray, 1966], to those with latitudinal dependence in surface properties [Fanale, 1976; Farmer and Doms, 1979; Zent et al., 1986], to those with longitudinal dependence [Paige, 1992; Mellon and Jakosky, 1993, 1995; Mellon et al., 1997], and, most recently, observed spatial and temporal variations in atmospheric humidity [Mellon et al., 2004; Schorghofer and Aharonson, 2005]. Comparison of these model predictions with the observations argues that

\footnotetext{
${ }^{1}$ Division of Geological and Planetary Sciences, California Institute of Technology, Pasadena, California, USA.

${ }^{2}$ Institute for Astronomy, University of Hawaii at Manoa, Honolulu, Hawaii, USA.
}

Copyright 2006 by the American Geophysical Union. 0148-0227/06/2005JE002636\$09.00 ground ice has adjusted to the atmospheric humidity owing to vapor diffusion through the regolith [Boynton et al., 2002; Mellon et al., 2004; Schorghofer and Aharonson, 2005]. Global maps of ice stability produced by these models assume a smooth, horizontal surface, that is, a "billiard ball" Mars. Here we evaluate the effect of surface roughness and find that slopes alter the global equilibrium ice distribution, and more generally, they allow ice to exist where previous global-scale models indicated it is unstable.

[4] The stability of ice with respect to diffusion is determined by vapor densities. For an atmospheric water vapor density $\rho_{v}$ and a saturation vapor density $\rho_{s v}$, ice is stable in equilibrium when densities balance [Mellon and Jakosky, 1993; Schorghofer and Aharonson, 2005],

$$
\bar{\rho}_{v}(\text { surface })=\bar{\rho}_{s v}(\text { at depth }) .
$$

Overbars indicate time averages. The mean annual vapor density $\rho_{v}$ is given by the atmospheric humidity near the surface, and $\rho_{s v}$ is a function purely of subsurface temperature. Thermal models of the surface and subsurface therefore provide the needed information for the stability condition. They provide not only the burial depth of an ice table in equilibrium, but the condition for perennial stability at any depth, that is, whether or not there is (or would be) a net loss of ice to the atmosphere. If $\bar{\rho}_{v}$ on the surface is equal to or exceeds $\bar{\rho}_{s v}$ at any depth, a patch of ice will remain stable or grow. Our model calculations are restricted to a two layer model, where a dry layer (free of perennial ice) 


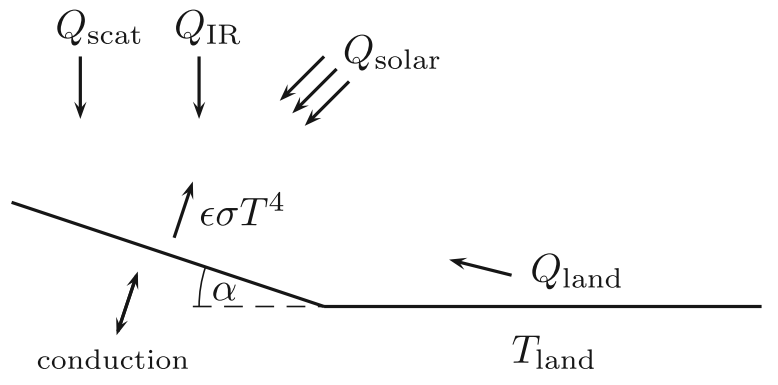

Figure 1. Contributions to the heat balance on a slope with surface temperature $T$.

overlies an ice rich layer (with uniform ice content). They take into account the thermal conduction by ice.

[5] The next section describes the thermal model used to determine temperature and ice stability on slopes. Section 3 describes the derivation of the distribution of slopes on Mars from altimeter measurements, and how these measurements are extrapolated to smaller baselines. The calculation of ice stability as a function of slope in section 2 and the histograms of slopes from section 3 are combined in section 4 to estimate the ground ice distribution on a rough Mars. The implications of the results for the Martian water history and comparison with geomorphological evidence are discussed in the last section.

\section{Ground Ice "Slope Effect"}

[6] Local slopes alter surface and subsurface temperatures substantially because of the difference in incidence sunlight and outgoing radiation. For example, data from the Thermal Emission Imaging System (THEMIS) on board Mars Odyssey reveals strong temperature variations over small distances. Spatial temperature heterogeneity is also evident in seasonal carbon dioxide ice, observed to adhere to topographic slopes [James et al., 2001; Schorghofer and Edgett, 2006]. Temperatures, in turn, control the stability of ground ice. Ice remains in the subsurface indefinitely when temperatures are sufficiently cold. As a result, local slopes can alter the ground ice distribution. Areas where ground ice is not otherwise stable may become favorable sites of deposition (the reverse is also possible).

\subsection{Energy Balance on a Slope}

[7] In order to examine this effect, a one-dimensional vertical model of surface and subsurface temperatures is used. The model is based on that of Kieffer et al. [1977] and follows closely the implementation of Schorghofer and Aharonson [2005], where further details and comparisons to previous thermal models are also given.

[8] We have improved the slope implementation and the atmospheric component of the model, and in the following provide a self consistent description of the energy balance on a slope. The atmospheric component and the slope implementation closely resemble the models by Kreslavsky and Head [2005] and Schorghofer and Edgett [2006].

[9] The heat balance on the surface is

$$
Q(\alpha)+\left.k \frac{\partial T}{\partial z}\right|_{z=0}=\epsilon \sigma T^{4}+L_{\mathrm{CO}_{2}} \frac{\partial m_{\mathrm{CO}_{2}}}{\partial t},
$$

with

$$
Q=Q_{\text {solar }}(\alpha)+Q_{\mathrm{IR}}(\alpha)+Q_{\text {scat }}(\alpha)+Q_{\text {land }}(\alpha) .
$$

$Q$ is the incoming radiation from the sun, atmosphere, and visible surfaces, $\alpha$ the slope angle, $T$ temperature, $z$ the vertical coordinate, $k$ the thermal conductivity, $\epsilon$ emissivity, $\sigma$ the Stefan-Boltzmann constant, $L_{\mathrm{CO}_{2}}$ the latent heat of $\mathrm{CO}_{2}, m_{\mathrm{CO}_{2}}$ the areal mass density of $\mathrm{CO}_{2}$, and $t$ time. The various contributions to $Q$ will be defined below. Figure 1 illustrates the contributions when no $\mathrm{CO}_{2}$ ice is present.

[10] A local slope has the effect of changing the incidence angle as well as reducing the fraction of cold sky to which the surface radiates. The elevation $\beta$ of the sun above a horizon is given in terms of geographic latitude $\lambda$, declination $\delta$ of the sun, and the hour angle $h$,

$$
\sin \beta=\cos \lambda \cos \delta \cos h+\sin \lambda \sin \delta .
$$

The coordinates of the sun are calculated using orbital elements from Allison and McEwen [2000]. The angle $\beta_{s}$ of the sun above a sloped surface is

$$
\sin \beta_{s}=\cos \alpha \sin \beta-\sin \alpha \cos \beta \cos (\Delta a),
$$

where $\Delta a$ is the difference between the azimuth of the sun and the azimuth of the topographic gradient. The sun is assumed to be below the horizon if either $\sin \beta<0$ (horizontal at infinity) or $\sin \beta_{s}<0$ (self shadowing of slope).

[11] The direct solar insolation is

$$
Q_{\text {solar }}=\frac{S_{0}}{R^{2}}(1-A)(1-f)^{1 / \max (\sin \beta, 0.04)} \sin \beta_{s},
$$

where $S_{0}$ is the solar constant, $R$ the distance from the sun in $\mathrm{AU}, A$ the albedo, and the factor $f$ due to the extinction in the atmosphere. The nadir optical depth of the atmosphere is $-\ln (1-f)$. The length of the path through the atmosphere is proportional to $1 / \sin \beta$ and the transmission is taken to be exponential in this path length. The maximum atmospheric path length $\ell_{\max }$ is limited due to the radius of curvature $R$ of the planet, $H_{a} / \ell_{\max } \approx$ $\sqrt{H_{a} / 2 R} \approx 0.04$, where $H_{a}$ is the scale height of the atmosphere.

[12] Atmospheric emission is approximated by a fraction $f_{\mathrm{IR}}$ of noontime insolation and is kept constant throughout a solar day [Kieffer et al., 1977],

$$
Q_{\mathrm{IR}}=f_{\mathrm{IR}} \frac{S_{0}}{R^{2}} \cos ^{2}\left(\frac{\alpha}{2}\right) \sin \beta_{\text {noon }} .
$$

The slope dependent term $\cos ^{2}(\alpha / 2)$ for the IR absorption takes into account that a tilted surface facing a horizontal horizon sees only a restricted portion of the sky. During polar winter the atmospheric emission vanishes in our model.

[13] In addition, there is scattered light when $\sin \beta>0$, which is approximated by 
Table 1. Model Parameters

\begin{tabular}{ll}
\hline \multicolumn{1}{c}{ Parameter } & \multicolumn{1}{c}{ Description } \\
\hline $\begin{array}{l}\text { Atmospheric } \\
\text { parameters }\end{array}$ & $f_{\mathrm{IR}}=0.04^{\mathrm{a}}, f_{\text {scat }}=0.02$ \\
Dry regolith properties & $c=800 \mathrm{~J} \mathrm{~kg}^{-1} \mathrm{~K}^{-1}, \epsilon=1$ \\
& $\rho$ varies as in work of Mellon and Jakosky [1993] \\
& $k$ is derived from thermal inertia \\
$\mathrm{CO}_{2}$ frost properties & $L_{\mathrm{CO}_{2}}=6 \times 10^{5} \mathrm{~J} \mathrm{~kg}^{-1}, A=0.65, \epsilon=1$ \\
& $\mathrm{Northern} \mathrm{Hemisphere} \mathrm{frost} \mathrm{point} 147 \mathrm{~K}$ \\
& Southern Hemisphere frost point $143 \mathrm{~K}$ \\
$\mathrm{H}_{2} \mathrm{O}$ ice properties & $c=1540 \mathrm{~J} \mathrm{~kg}^{-1} \mathrm{~K}^{-1}$ \\
& $\rho=927 \mathrm{~kg} \mathrm{~m}^{-3}, k=3.2 \mathrm{~W} \mathrm{~m}^{-1} \mathrm{~K}^{-1}$ \\
\hline
\end{tabular}

${ }^{\mathrm{a}} \mathrm{A} 2 \%$ contribution is utilized by Kieffer et al. [1977] and commonly used afterward. Haberle and Jakosky [1991] compared this approximation with atmospheric models, and found that for a clear atmosphere the infrared emission is approximately twice as large than that estimated from the $2 \%$ approximation. Hence we choose $f_{\mathrm{IR}}=0.04$.

$$
Q_{\text {scat }}=\frac{1}{2} f_{\text {scat }} \frac{S_{0}}{R^{2}} \cos ^{2}\left(\frac{\alpha}{2}\right) .
$$

Half of the scattered light is assumed to be lost to space.

[14] A patch of ground reemits radiation in all directions, but receives additional heat from surfaces in its field of view. This emission is weighted according to the incidence angle $i$ and integrated over the spherical angle $\Omega$ subtended by the visible land surfaces. If we imagine a horizontal surface at uniform temperature $T_{\text {land }}$ [Kreslavsky and Head, 2005],

$$
Q_{\text {land }}=\epsilon_{\text {land }} \sigma T_{\text {land }}^{4} \int \cos i d \Omega=\sin ^{2}\left(\frac{\alpha}{2}\right) \epsilon_{\text {land }} \sigma T_{\text {land }}^{4} .
$$

If $T_{\text {land }}=T$ and $\epsilon_{\text {land }}=\epsilon$, then this term can be brought to the right-hand side of equation (2), leading to an effective emissivity of $\epsilon \cos ^{2}(\alpha / 2)$, but we do not make this approximation.

[15] When the surface temperature reaches the sublimation temperature of $\mathrm{CO}_{2}$, then $\mathrm{CO}_{2}$ accumulates, the albedo increases, and the surface temperature remains constant until all the $\mathrm{CO}_{2}$ has disappeared again. While it is present, heat balance is accomplished by $\mathrm{CO}_{2}$ accumulation and sublimation.

[16] The equation of heat conduction is solved in the subsurface with a semi-implicit (unconditionally stable) Crank-Nicholson scheme on a grid with spatially varying spacings,

$$
\rho c \frac{\partial T}{\partial t}=\frac{\partial}{\partial z}\left(k \frac{\partial T}{\partial z}\right)
$$

The presence of ground ice changes the thermal conductivity $k$, heat capacity $c$, and density $\rho$ of the ground, and the equilibrium ice depth is solved for iteratively. Several model parameters are listed in Table 1.

[17] Among the model parameters, few affect the annual mean temperature substantially. A contribution that is significant for steep slopes is thermal emission from other surfaces in the field of view. In Figure $2 \mathrm{a}$, the total incoming radiation $Q$ is plotted as a function of slope, and compared to the contribution from a nearby horizontal surface $Q_{\text {land }}$. For most slopes, solar radiation dominates, but steep polefacing slopes receive an important contribution from the surrounding land. Figure $2 \mathrm{~b}$ shows mean temperatures as a function of slope. Solid and dash lines differ in the way the thermal emission from horizontal surfaces to sloped surfaces is calculated. The dash line assumes the horizontal surface has the same temperature as the slope. The solid line uses a model with different temperatures on the slope and the horizontal surface. The latter temperature model is necessary for steep pole-facing (positive) slopes, for which the thermal emission from land surfaces contributes substantially to the energy input.

\subsection{Model Results}

[18] The results of the thermal modeling as a function of surface slope are summarized below. We consider first the effect of slope before turning to the global distribution.

[19] After model temperatures have equilibrated, vapor densities are computed from the last Mars year. The difference $\Delta \rho_{v}$ between the average vapor density on the surface and the saturation vapor density of any buried ice indicates stability. In the model, no supersaturation is allowed; that is, the humidity of the nighttime atmosphere is limited by the saturation vapor pressure.

[20] Figure 3 shows mean temperatures and equilibrium ice table depth as a function of slope, using thermal

(a)

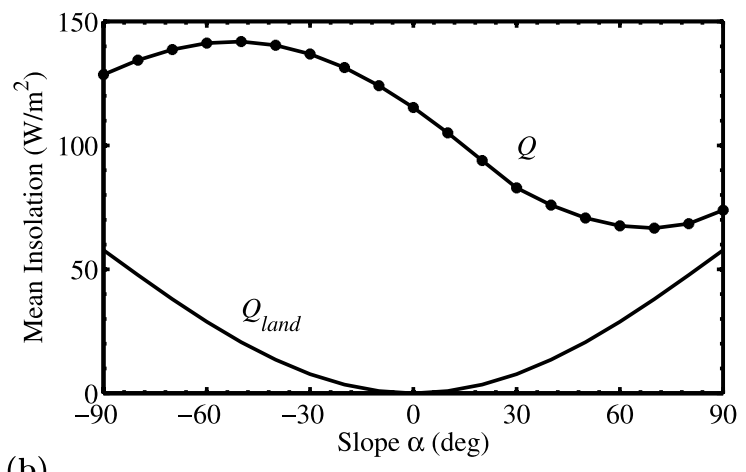

(b)

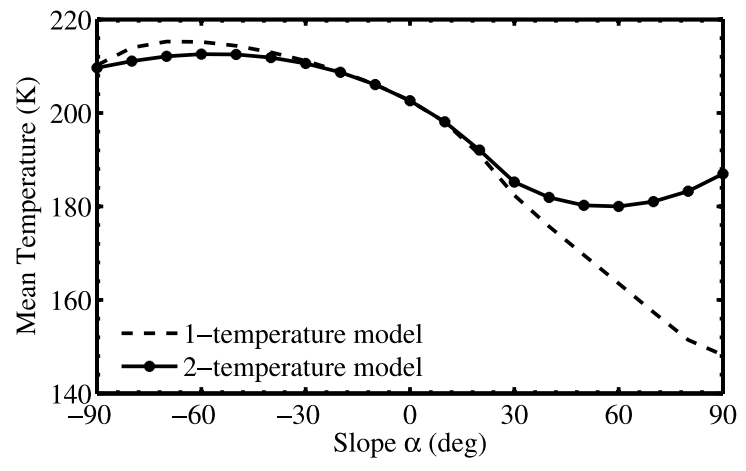

Figure 2. (a) Annual mean insolation $Q(\alpha)$ and the contribution of the thermal emission from land, $Q_{\text {land }}$ at a latitude of $35^{\circ} \mathrm{N}$. The albedo of the surface is $A=0.3$ and the thermal inertia $I=150 \mathrm{~J} \mathrm{~m}^{-2} \mathrm{~K}^{-1} \mathrm{~s}^{-1 / 2}$. Pole-facing slopes are shown as positive and equator-facing as negative. (b) Mean surface temperature as a function of slope. Two models which differ in their treatment of the emission from the horizontal surface are shown: in the first the horizontal surface is assumed to have the same temperature as the slope (dashed line), and in the second the horizontal surface temperature is solved for separately (solid line). 


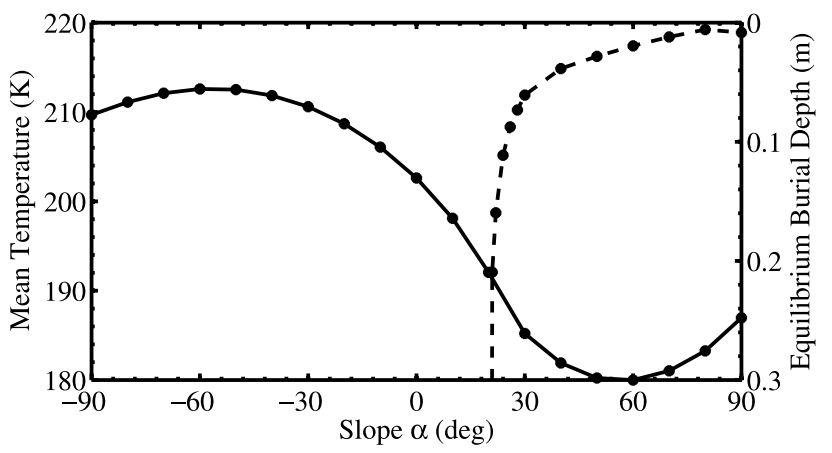

Figure 3. Mean temperatures (solid line) and equilibrium ice table depth (dashed line) as a function of slope at a latitude of $35^{\circ} \mathrm{N}$. Thermal parameters are as in Figure 2 and $T_{f}=200 \mathrm{~K}$. Pole-facing slopes are colder than equatorfacing slopes and accumulate or retain ice beyond a slope of about $20^{\circ}$. Mean temperatures need to be well below the frost point temperature for ice to be stable.

properties and latitude appropriate for the northern Olympus Mons aureole. At this site at $35^{\circ} \mathrm{N}$, ice is unstable on horizontal ground, but becomes stable beneath pole-facing slopes [Schorghofer and Aharonson, 2005]. Note that at this location, the coldest surfaces are not vertical but are $55-60^{\circ}$ pole-facing slopes.

[21] The equilibrium ice table is determined by first calculating the mean vapor density of saturated air five seasonal skin depths beneath the surface and the mean vapor density at the surface. If ice is stable, $\Delta \rho_{v} \leq 0$, then a bisection algorithm is employed to search for the equilibrium ice table depth. As seen in Figure 3, ground ice becomes stable only when temperatures are significantly below the frost point temperature $T_{f}$ of the daytime atmosphere (here $200 \mathrm{~K}$ ). This is chiefly attributable to nighttime saturation reducing the average humidity below its daytime value [Schorghofer and Aharonson, 2005].

[22] Surface temperatures at different slopes are coupled due to thermal emission. We consider emission from horizontal surfaces to slopes, but not in the other direction, because the angle subtended by a slope (e.g., the height of a crater rim) is typically small. The equilibrium ice table depth is first computed for a horizontal slope and then temperatures and ice table depths are obtained for all slopes larger than zero.

[23] Another example calculation is presented in Figure 4 for a horizontal surface (dashed line) and a pole-facing slope (solid line) as a function of latitude with constant thermal properties, again using parameters typical of the Olympus Mons aureole. Ice stability on pole-facing slopes extends approximately $16^{\circ}$ farther equatorward than on a horizontal surface. Still, ice is far from stable near equatorial latitudes even for this low thermal inertia.

[24] We now turn to global temperature calculations. These use, as inputs, the thermal inertia map of Putzig et al. [2005], the albedo map of Christensen et al. [2001], and partial surface pressures obtained from water column abundance maps by Smith [2002], all of which are derived from Thermal Emission Spectrometer (TES) measurements [Christensen et al., 2001]. Measurements of thermal inertia are based on diurnal temperature variations and are taken to represent the thermal inertia of the upper, dry layer. The diurnal skin depth is typically a few centimeters. The partial pressure of $\mathrm{H}_{2} \mathrm{O}$ at the surface is approximately proportional to the vapor column abundance. (Extraction of surface humidities is described in detail in Appendix $\mathrm{C}$ of Schorghofer and Aharonson [2005].) The surface partial pressure of $\mathrm{H}_{2} \mathrm{O}$ is longitude and latitude dependent, and has a global daytime average that corresponds to a frost point of $T_{f}=198 \mathrm{~K}$. The frost point temperature within $\pm 70^{\circ}$ latitude varies from $192-203 \mathrm{~K}$ depending on geographic location. These global calculations are computationally expensive, and were performed using a cluster of 146 processors dedicated for several weeks.

[25] Results of this model are shown in Figure 5, plotting the depths to a hypothetical ice table in equilibrium, beneath a $30^{\circ}$ slope at various azimuthal orientations. For example, the top left panel shows pole facing slopes in the Northern Hemisphere contain ice down to lower latitudes than does horizontal ground. Although these maps use the actual geographic variation of thermal inertia, albedo, and humidity, they do not show the actual ground ice distribution, because the slope is taken to be the same everywhere. The ice distribution is rather zonal, because latitude exerts a strong control on temperature. There is an approximate $30^{\circ}$ latitude difference between the stability boundary of northand south-facing models.

\section{Rough Mars: Slope Statistics Derived From Altimetry}

\subsection{Adirectional Slopes}

[26] Elevation measurements are available from the Mars Orbiter Laser Altimeter (MOLA) at a separation of $\sim 300 \mathrm{~m}$ along track, and typically $1-2 \mathrm{~km}$ across tracks. In order to take advantage of the higher resolution along track, we

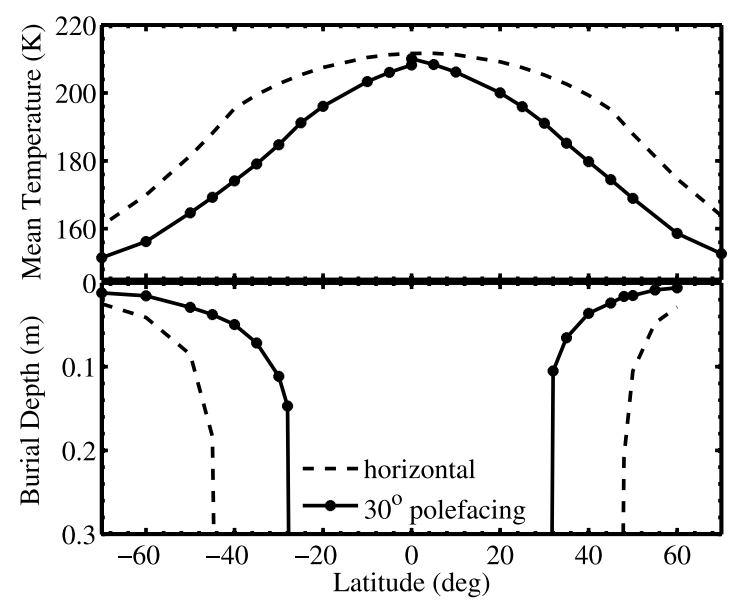

Figure 4. Mean temperatures and equilibrium depth to ice table for a horizontal surface (dashed line) and a polefacing slope (solid line) as a function of latitude with constant thermal properties, $A=0.3, I=150 \mathrm{~J} \mathrm{~m}^{-2} \mathrm{~K}^{-1} \mathrm{~s}^{-1 / 2}$, $T_{f}=200 \mathrm{~K}, \alpha=30^{\circ}$. Ice is far from stable near equatorial latitudes. The small discontinuity in the surface temperature at the equator is due to the change from south-facing to north-facing slopes. 

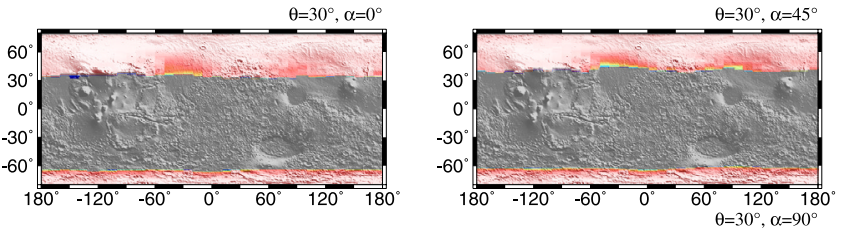

$\begin{array}{llllllllllllll}0.0 & 0.1 & 0.2 & 0.3 & 0.4 & 0.5 & 0.6 & 0.7 & 0.8 & 0.9 & 1.0\end{array}$ Ice Table Depth (m)
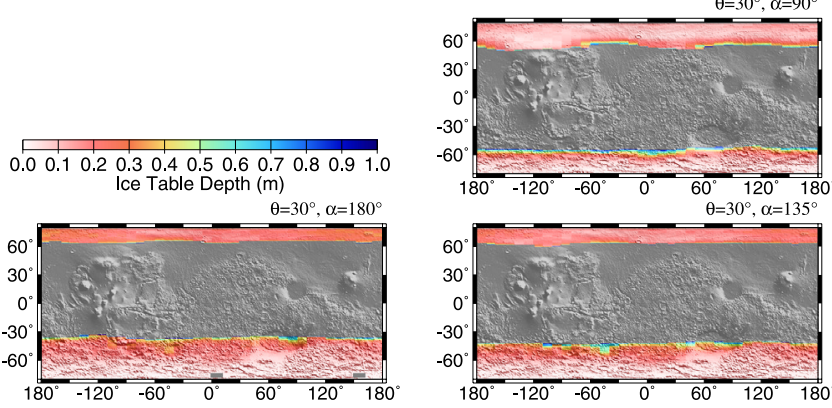

Figure 5. Maps showing depth to the ice table in equilibrium beneath a slope of $30^{\circ}$ with azimuths of $0^{\circ}$ (north facing), $45^{\circ}$ (northeast facing), up to $180^{\circ}$ (south facing) in intervals of $45^{\circ}$. The position of each panel corresponds to the direction of the downhill gradient. The computation was performed for all eight azimuths, but only the east facing slopes are shown because the results were nearly exactly symmetric in the east-west directions.

extract point-to-point topographic slopes from transects rather than from the gridded data. Given this directional slope distribution $\tilde{F}(p)$, we wish to derive the adirectional slope distribution $F(s)$. Here we perform this transformation, analytically and numerically.

[27] The directional and adirectional slopes $p$ and $s$ are related by

$$
p=s \cos \theta
$$

where $\theta$ measures the angle between the direction of the topographic gradient and the direction of the spacecraft ground track, and $s=\tan \alpha$. The probability distribution functions are related by an integration over a $\delta$-function,

$$
F(s)=\iint \tilde{F}(p, \theta) \delta\left(s-\frac{p}{\cos \theta}\right) d p d \theta
$$

or alternatively,

$$
\tilde{F}(p)=\iint F(s, \theta) \delta(p-s \cos \theta) d s d \theta .
$$

Integrals over $\theta$ are implicit to be from 0 to $2 \pi$, those over $p$ are from $-\infty$ to $\infty$, and over $s$ from 0 to $\infty$. Performing the $\theta$ integration with the assumption of a statistically isotropic slope distribution $F(s, \theta)=F(s) /(2 \pi)$, results in

$$
\tilde{F}(p)=\frac{1}{\pi} \int_{|p|}^{\infty} \frac{F(s)}{\sqrt{s^{2}-p^{2}}} d s .
$$

The lower integration limits are due to the fact that only values of $s \geq|p|$ contribute to any observed $p$. A factor of 2 was introduced because every value of $s$ may be obtained from 2 values of $\theta$. The integral in (14) may be performed analytically for only a few functions. For example, in the special case,

$$
F(s)=s e^{-s^{2} / 2},
$$

the integral leads to a Gaussian distribution in $p$,

$$
\tilde{F}(p)=\frac{1}{\sqrt{2 \pi}} e^{-p^{2} / 2}
$$

The ratio of the root-mean-square (RMS) values is, in this case,

$$
\left(\frac{\int s^{2} F(s) d s}{\int p^{2} \tilde{F}(p) d p}\right)^{1 / 2}=\sqrt{2} .
$$

This ratio is always larger than one because the slope along a transect can never be greater than in the direction of steepest descent.

[28] For real data, we discretize the integral in (14) and treat it as a matrix equation with

$$
\tilde{F}\left(p_{i}\right)=\sum_{j} M_{i j} F\left(s_{j}\right),
$$

where

$$
M_{i j}= \begin{cases}\frac{1}{\pi} \frac{1}{\sqrt{s_{j}^{2}-p_{i}^{2}}} \delta s, & s_{j}>\left|p_{i}\right|, \\ 0 & \text { otherwise. }\end{cases}
$$

[29] Here $\delta s$ denotes the bin width. Standard linear algebra techniques now allow solving for $F\left(s_{j}\right)$. Care must be taken to avoid choosing $s_{j}=\left|p_{i}\right|$, since the denominator vanishes. The integral may be estimated more robustly by assuming $F(s)$ is constant within each integration interval (or histogram bin when operating on discrete data). Utilizing $\int 1 / \sqrt{s^{2}-p^{2}} d s=\log (s+$ $\left.\sqrt{s^{2}-p^{2}}\right)$ to within an integration constant, we obtain

$$
M_{i j}=\frac{1}{\pi} \begin{cases}\left.\log \left(s+\sqrt{s^{2}-p_{i}^{2}}\right)\right|_{s_{j}-\delta s / 2} ^{s_{j}+\delta s / 2} & s_{j}>\left|p_{i}\right|, \\ \left.\log \left(s+\sqrt{s^{2}-p_{i}^{2}}\right)\right|_{s_{j}} ^{s_{j}+\delta s / 2} & s_{j}=\left|p_{i}\right|, \\ 0 & s_{j}<\left|p_{i}\right| .\end{cases}
$$

[30] Using this technique, probability distribution functions (PDFs) derived from MOLA data are shown in Figure 6. Note in this case, the RMS of $s$ is roughly 1.4 times that of $p$, as would be expected for a Gaussian slope distribution (equation (17)). Pole facing and equator facing slopes are computed separately, so each curve is actually composed of two that are nearly identical (a slight but meaningful asymmetry exists for steep slopes, first 


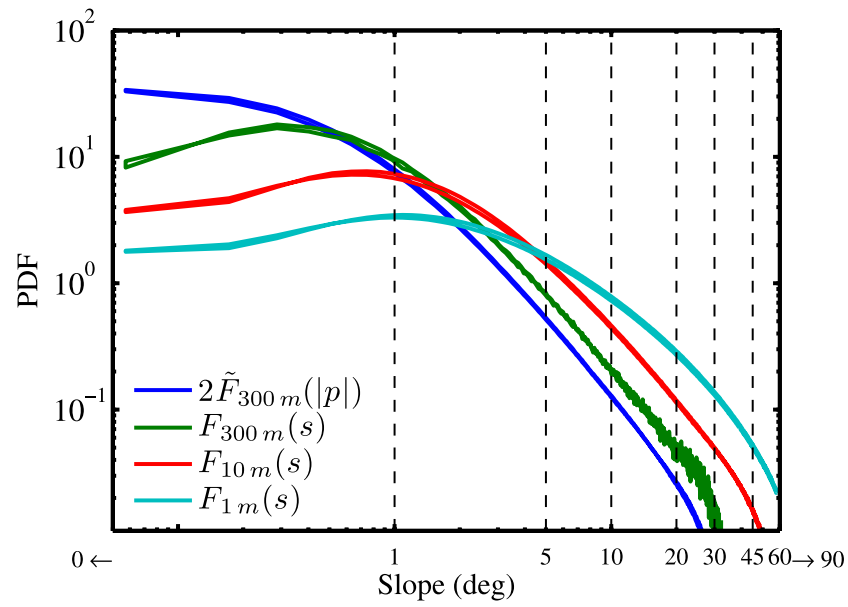

Figure 6. Slope histograms showing the PDF of the original MOLA measured one-dimensional (1-D) slopes $\tilde{F}(p)$, the derived distribution of 2-D gradients $F(s)$, and this distribution extrapolated to $10 \mathrm{~m}$ and $1 \mathrm{~m}$ scales. All histograms are normalized to unit area, but $\tilde{F}(p)$ is multiplied by 2 for comparison. Dashed lines indicate the bin boundaries used for convolution with the ice prediction.

reported by Kreslavsky and Head [2003]). The curve labeled $\tilde{F}_{300 \mathrm{~m}}(p)$ shows the directional point-to-point slopes derived from MOLA, and $F_{300 \mathrm{~m}}(s)$ shows the derived adirectional gradient magnitudes.

\subsection{Slope Scale Dependence}

[31] In order to relax the "billiard ball" Mars assumption we require an estimate of the real slope distribution. Selecting the length scale of slopes most relevant for ice accumulation, and quantifying the slope distribution at that length scale on Mars are both complicated issues. We expect fluctuations in the ice table depth to occur over spatial scales comparable to the thermal skin depth, i.e., decimeters. The surface roughness of Mars at this wavelength is not known on a global scale. The approach chosen here is one in which Mars Orbiter Laser Altimeter (MOLA) point-to-point slopes [Aharonson et al., 2001] are extrapolated to the expected relevant length scale of $1 \mathrm{~m}$. Partially supporting this extrapolation are previous attempts to compare slopes at multiple scales derived from orbital and lander images [Aharonson, 2002; Golombek et al., 2003]. The extrapolation is likely to be more secure from scales of a few hundreds of meters down to tens of meters than over the last decade of extrapolation, from $10 \mathrm{~m}$ down to $1 \mathrm{~m}$.

[32] The structure function $\zeta$ describes the autocorrelation of the elevation $z$ over a baseline $l$, and is defined by

$$
\zeta(l)=\left\langle|z(x)-z(x+l)|^{2}\right\rangle_{x}^{1 / 2},
$$

where \langle\rangle$_{x}$ denotes spatial averaging, in our case along the spacecraft ground-track. If $\zeta(l)$ can be approximated with a power law, then the exponent $H$ is called the Hurst exponent, and is defined by

$$
\zeta(l)=\zeta_{0} l^{H} .
$$

The exponent of the RMS slope $s_{\mathrm{RMS}}$ as a function of baseline is reduced by 1 , as can be seen from

$$
s_{\mathrm{RMS}}(l)=\left\langle\left|\frac{z(x)-z(x+l)}{l}\right|^{2}\right\rangle_{x}^{1 / 2}=\zeta_{0} l^{H-1} .
$$

[33] When using real data, we choose to measure median absolute slopes instead of RMS slopes, because the former estimate is more robust, i.e., less sensitive to outliers in the slope distribution [Aharonson et al., 2001]. We can derive a Hurst exponent from median absolute slopes $s_{\text {MAS }}$, at two baselines.

[34] Consider elevation measurements separated by $300 \mathrm{~m}, 600 \mathrm{~m}$, and $1.2 \mathrm{~km}$, i.e., 1, 2, and 4 MOLA shot measurements, respectively. Using the $300 \mathrm{~m}$ with $600 \mathrm{~m}$ slopes and $600 \mathrm{~m}$ with $1.2 \mathrm{~km}$ slopes, two independent values for $H$ are derived, and their average computed. The global data, area-weighted histogram of these estimates for $H$ are shown in Figure 7a. The estimates for $H$ over this (a)

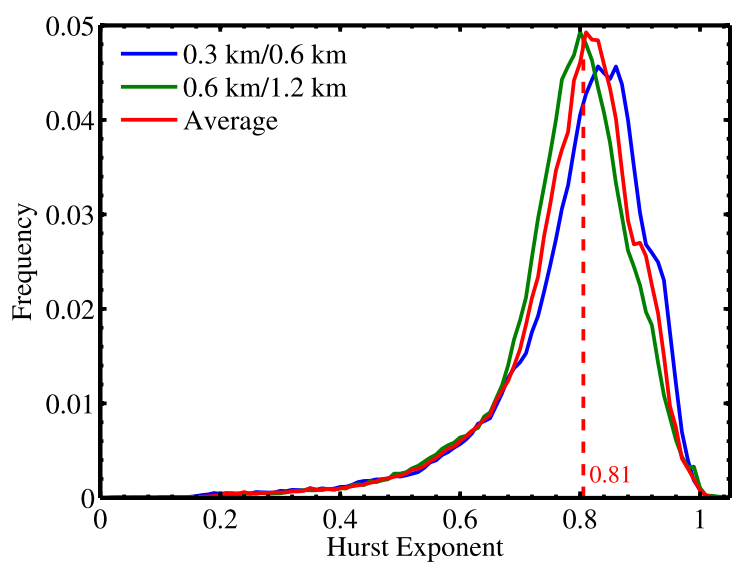

(b)

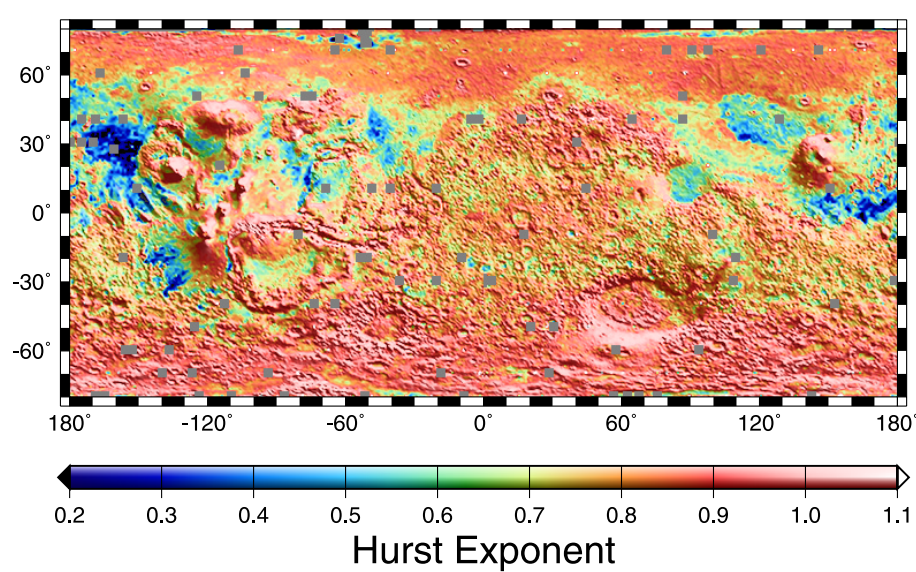

Figure 7. (a) Equal-area histogram of various estimates of Hurst exponent derived from MOLA $0.3 \mathrm{~km}$, $0.6 \mathrm{~km}$ and $1.2 \mathrm{~km}$ slopes. (b) Map view of Hurst exponent. 
(a)

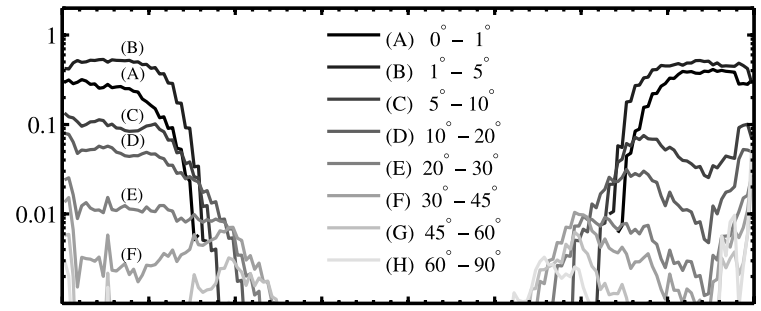

(b)

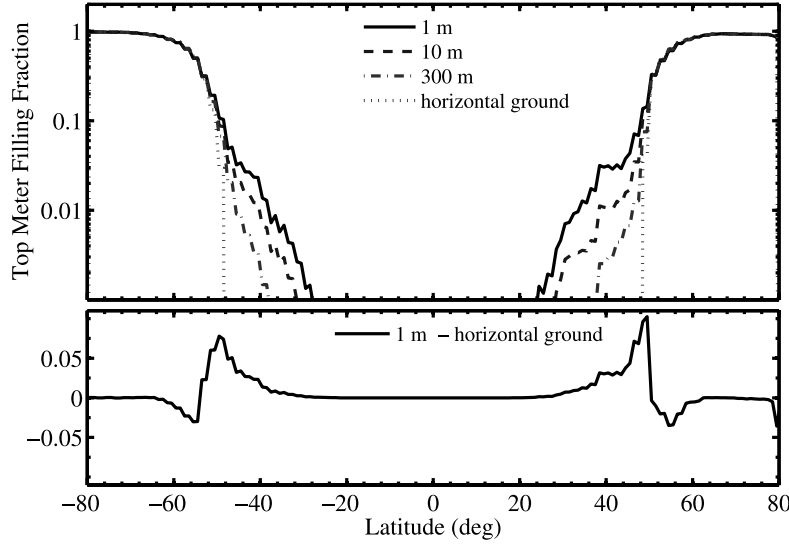

Figure 8. Equilibrium ice filling fraction in the top one meter of regolith, as a function of latitude averaged over the eight azimuthal slope directions and over all longitudes. (a) The contribution of each slope bin to the ice content is shown. At the high latitudes, ice is stable beneath shallow slopes; at lower latitudes the contribution of steeper slopes grows, increasing their relative importance. (b) Roughness models with various baselines are compared with each other and with horizontal ground. (c) The difference in ice contents beneath horizontal ground and a 1-m roughness model.

scale have a similar range, with their means differing by only $\sim 2 \%$. The global median Hurst exponent is 0.81 . The averaged $H$ is shown in map form in Figure $7 \mathrm{~b}$. The typical value of $H=0.81$ would give roughly a factor of 3 enhancement in roughness from $300 \mathrm{~m}$ to $1 \mathrm{~m}$. For lower $H$, such as typical in Amazonis Planitia for example, the enhancement factor is greater.

[35] Figure 6 shows the slope distributions using local estimates of the Hurst exponent, extrapolated to an intermediate $10 \mathrm{~m}$ scale $F_{10 \mathrm{~m}}(s)$, and to the desired $1 \mathrm{~m}$ scale $F_{1 \mathrm{~m}}(s)$. Slopes steeper than a few degrees become more abundant at the expense of shallower slopes. In particular, slopes steeper than $10^{\circ}$ are significant contributors to the distribution at the $1 \mathrm{~m}$ scale.

\section{Ground Ice Beneath Real Topography}

[36] Having computed the dependence of the ice table on gradient and azimuth (section 2), and the extrapolated gradient distribution (section 3), we now turn to convolving these estimates to predict a distribution of ground ice that includes topographic control.

[37] The slope distribution is represented by bins delimited by the slopes $\{0,1,5,10,20,30,45,60,90\}^{\circ}$, where within each bin there are eight azimuthal bins. The total ice fraction is computed by trapezoidal integration of the azimuthally averaged ice fraction at each slope bin, multiplied by the fraction of surface at this slope. Averaging over the azimuthal bins is justified for an isotropic slope distribution. While a small slope asymmetry exists [Kreslavsky and Head, 2003], this effect is negligible here since it modifies only a small fraction, on order of a few percent, of the slopes.

[38] Results are reported in terms of top meter filling fraction, or ice fraction, defined as the fraction of space not occupied by regolith (pore space) that is filled with ice. This filling fraction varies from 0 (no ice within the top meter) to 1 (ice-filled layer extends all the way to the surface everywhere). While the absolute amount of ground ice depends directly on the ice content of the ice-filled layer, the filling fraction is a more general quantity. Although thermal conduction affects equilibrium burial depths, the variations are small for ice contents of more than tens of percents [Schorghofer and Aharonson, 2005]. Filling fractions for ice contents higher than the $40 \%$ assumed in the thermal model would be nearly the same. Another quantity used to represent our results is the top meter areal fraction. It gives the fraction of area where ice is stable, independent of burial depth, except that ice buried by more than $1 \mathrm{~m}$ is ignored.

[39] The zonally averaged ice fractions for roughness models with various baselines are shown in Figure 8. Figure 8a shows the contribution to the total ice content of each slope bin. The role of steep slopes is seen to increase in importance with decreasing latitude. The sum of these curves is shown in the 1-m curve of Figure $8 \mathrm{~b}$, where a comparison is made among the results for horizontal ground, slopes without extrapolation $(300 \mathrm{~m})$, partial extrapolation $(10 \mathrm{~m})$, and the final extrapolation $(1 \mathrm{~m})$. The horizontal ground zonal average is similar to that originally derived by Farmer and Doms [1979]. The difference between horizontal ground and the 1-m roughness model shown in Figure 8c shows a small decrease in ice content at the high latitudes where ice is stable beneath horizontal ground, and an increase in lower latitudes where ice was previously unstable.

[40] The slope extrapolation and convolution is performed using local parameters at each spatial location on a $10^{\circ}$ in longitude by $2^{\circ}$ in latitude grid. The results in map form are shown in Figure 9. In Figure 9a the slope is assumed to be 0 , and the ice distribution mirrors that of previous horizontal ground models [Mellon et al., 2004; Schorghofer and Aharonson, 2005]. We find ice is stable at latitudes poleward of $\sim 50^{\circ}$, and transitions abruptly to being unstable over 1 or 2 computational latitude grid points. Figure $9 \mathrm{~b}$ shows the filling fraction using the corrected gradient distribution $F_{300 \mathrm{~m}}(s)$, but with no extrapolation from the intrinsic $300 \mathrm{~m}$ MOLA scale. In this map, ground ice extends further equatorward with several percent filling fraction down to $\sim 38^{\circ}$ latitude. The ice content shows variability according to surface roughness. This estimate may be considered a lower bound as it involves no extrapolation of the slope distribution. Note the logarithmic axis which emphasizes small ice contents. Figure 9c shows the resulting ice distribution using the extrapolated slope distribution $F_{1 \mathrm{~m}}(s)$. Local thermal and topographic parameters of the surface extent the ice distribution down to $\sim 24^{\circ}$ latitude, with enhanced 
(a)

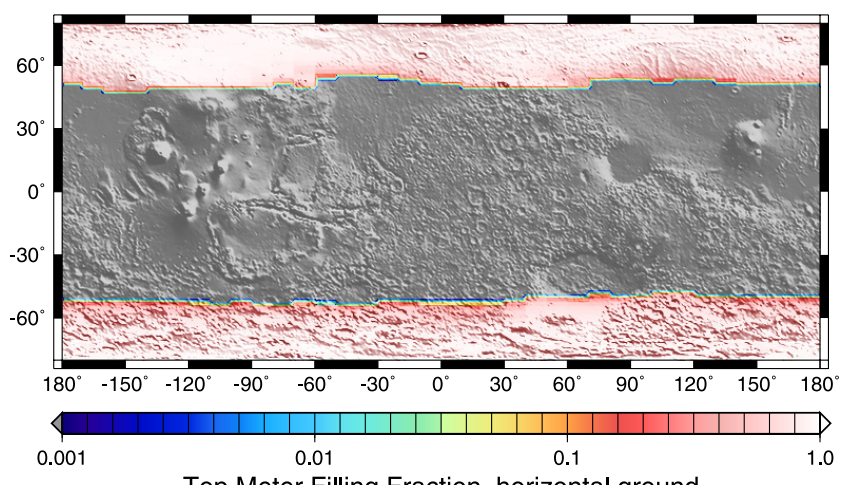

(c)

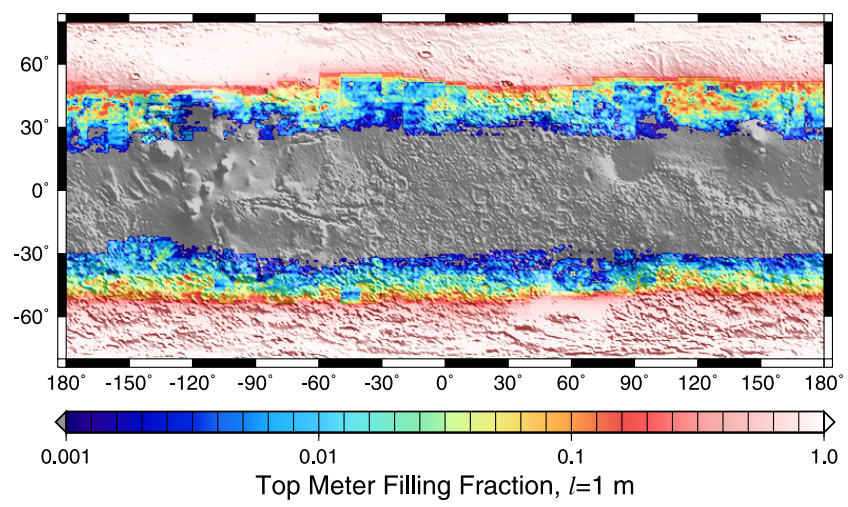

(e)

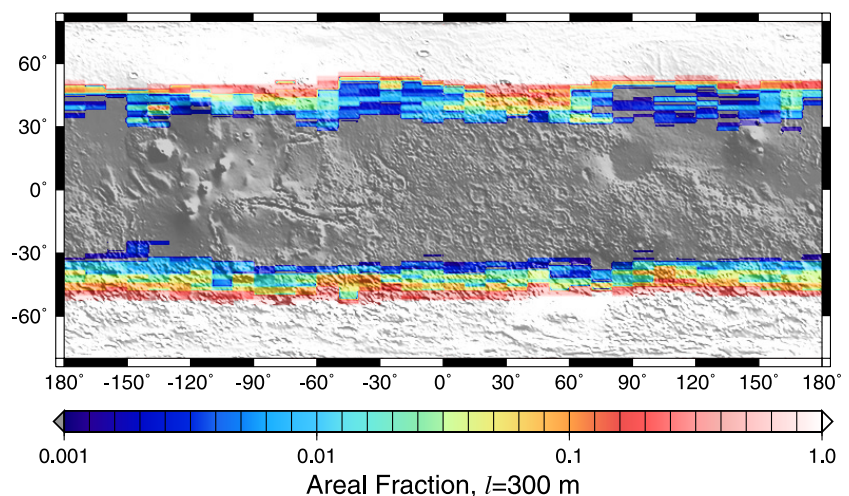

(b)

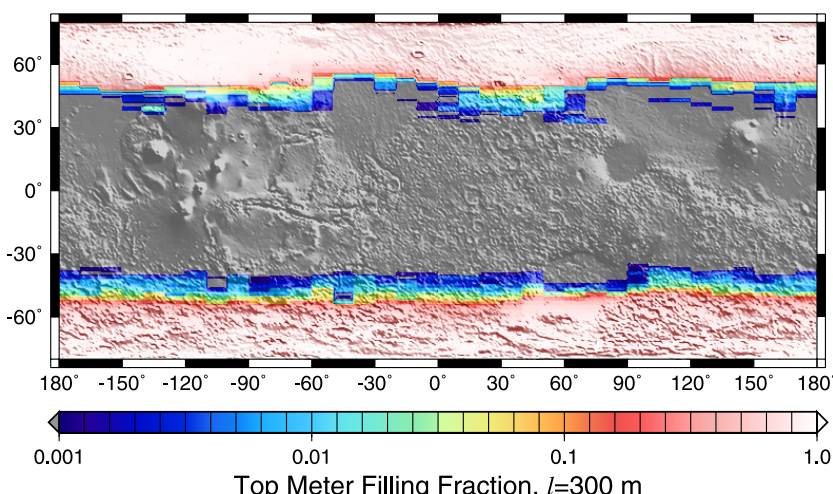

(d)

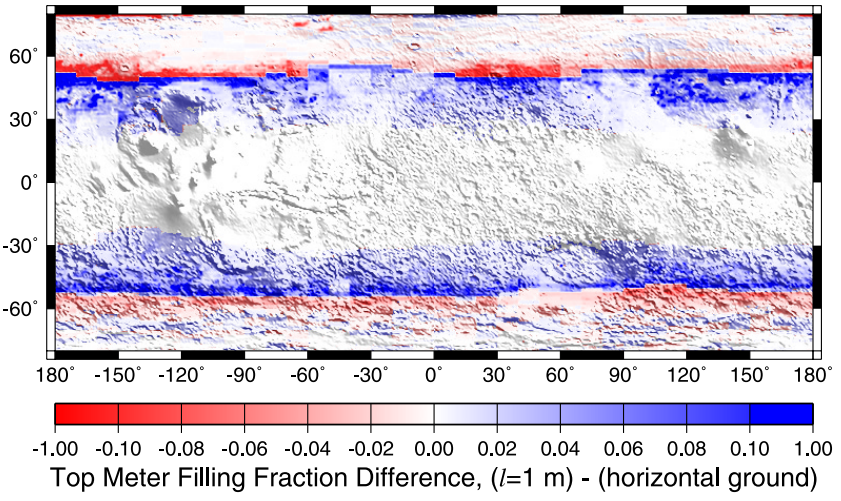

(f)

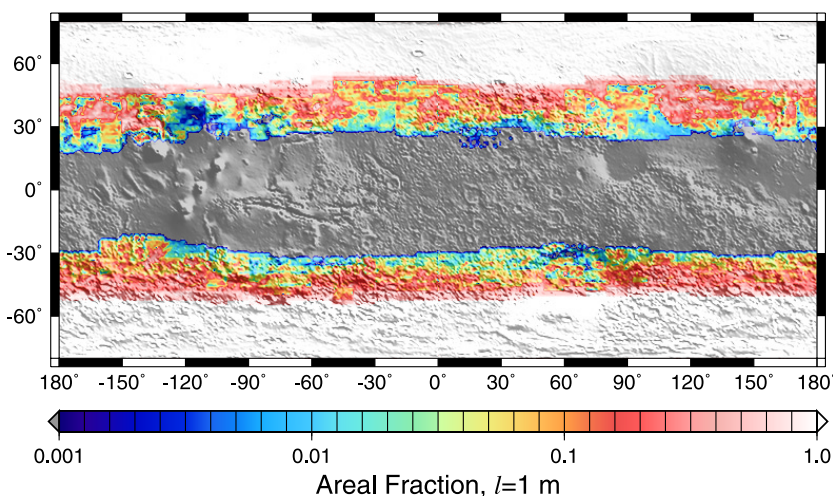

Figure 9. Maps showing estimated ice filling fraction in the top $1 \mathrm{~m}$ of regolith. (a) Horizontal ground model. (b) Assumed MOLA 300-m slopes, corrected for directionality. (c) Slope distribution extrapolated to 1-m scale. (d) Difference between extrapolated and horizontal ground calculations. (e) Areal fraction of ground where subsurface ice is expected with assumptions as in Figure 9b. (f) Ice areal fraction with assumptions as in Figure 9c. All models assume an isotropic slope distribution.

quantities in excess of $10 \%$ beneath rough areas, such as the Olympus Mons aureole deposits and variations associated with features such as Hecates Tholus and Hellas basin. The transition from ice rich to ice free ground now occurs over a substantial latitudinal band of about $30^{\circ}$. The difference between this and the horizontal ground models is shown in Figure 9d. It is clear that the model which includes surface roughness predicts both an increase in ice content relative to horizontal ground (in midlatitudes, where ice was previously unstable), as well as a decrease (in high latitudes, where the ice table was previously shallow). Figures 9e and 9f are analogous to Figures 9b and $9 \mathrm{c}$, but show the fraction of surface area where ice is stable.

\section{Discussion}

[41] Neutrons emitted from the subsurface are used to determine the ground hydrogen content from orbit. Neutron counts vary nonlinearly with ice fraction. 
Feldman et al. [2004] provide a calibration of neutrons counts versus mass fraction of water equivalent hydrogen (WEH). For example, 4 epithermal neutrons per second correspond to a uniform layer of $13 \% \mathrm{WEH}$, but could also result when half the area has 99\% WEH and the other half only $5 \% \mathrm{WEH}$, averaging to $52 \%$. Hence a geographically heterogeneous ground ice distribution reduces the epithermal neutron flux to a lesser degree than the same amount of ice uniformly distributed, impeding the detection of ice in the former case. Mass fractions shown in Figure 9 of order a few percent in the low latitudes may therefore escape detection by neutrons observed by Mars Odyssey. Overall, the results of the model presented here agree well with the observed hydrogen abundance in high- and midlatitudes from the Odyssey Gamma Ray Spectrometer [Boynton et al., 2002] and Neutron Detector [Feldman et al., 2004], although additional analysis must be performed to deconvolve the instruments' spatial response function for detailed comparisons. While the slope dependent thermal stability model predicts ice down to lower latitudes, it does not predict stable ice at the distribution of equatorial locations were elevated hydrogen abundances (of approximately $10 \%$ water equivalence) are observed.

[42] Geomorphological evidence for the presence of low-latitude subsurface ice has been reported. Rifkin and Mustard [2001] and Head et al. [2003] identified dissected mantle terrain and interpreted a high degree of volatile loss. Mikovich and Head [2003] interpret glacial ice on the northwestern slope of the basal escarpment of Olympus Mons. Hauber et al. [2005] cite evidence for glacial activity at Hecates Tholus. These regions, the dissected mantle terrain, the northwest slopes of the Olympus Mons aureole, and Hecates Tholus are within the areas of presently stable ice in Figures $9 \mathrm{c}$ and $9 \mathrm{f}$. The observed ice may be the result of remnant glaciation during previous obliquity cycles [Head et al., 2003] and its survival attributed to low vapor diffusivity of the overlying soil layer. Slopes provide an alternative explanation for the survival of subsurface ice, and perhaps even for the emplacement of some deposits. In this scenario, the ice adheres strongly to slopes and has a latitude dependence. Although not computed here, higher past obliquity would certainly extend stability to even lower latitudes. The slope effect computed here does not suffice to stabilize ice on the flanks of other Tharsis volcanoes, the near-equatorial Ascraeus, Pavonis and Arsia Mons; any ice previously deposited there should either have sublimated or be sublimating and diffusing out today, or else be protected by a diffusion barrier.

[43] We also find that the equilibrium ice content at the high latitudes depends only weakly on topographic roughness. High volume fractions of ice near polar latitudes supports an atmospheric condensation origin for the ground ice [Boynton et al., 2002], rather than introduction into the ground by vapor diffusion.

[44] Independent of the geomorphological evidence, the stability of ice on pole-facing slopes at present-day conditions leads to the expectation that ice was present not only in the recent past but can be found at these locations also today. Perhaps most importantly, areas in the midlatitudes with high surface roughness that have previously been predicted to be ice free are predicted to contain quantities of ice that may be accessible in the future.

[45] Acknowledgment. We wish to thank Misha Kreslavsky and Re'em Sari for helpful discussions. O. A. was supported by the NASA Mars Data Analysis Program. N. S. was supported by the NASA Astrobiology Institute under Cooperative Agreement NNA04CC08A issued through the Office of Space Science.

\section{References}

Aharonson, O. (2002), The surface of Mars: Morphology and process, Ph.D. thesis, Mass. Inst. of Technol., Cambridge.

Aharonson, O., M. T. Zuber, and D. H. Rothman (2001), Statistics of Mars' topography from MOLA: Slopes, correlations and physical models, J. Geophys. Res., 106, 23,723-23,735.

Allison, M., and M. McEwen (2000), A post-Pathfinder evaluation of areocentric solar coordinates with improved timing recipes for Mars seasonal diurnal climate studies, Planet. Space Sci., 48, 215-235.

Boynton, W. V., et al. (2002), Distribution of hydrogen in the near surface of Mars: Evidence for subsurface ice deposits, Science, 297, 81-85.

Christensen, P. R., et al. (2001), Mars Global Surveyor Thermal Emission Spectrometer experiment: Investigation description and surface science results, J. Geophys. Res., 106, 23,823-23,871.

Fanale, F. P. (1976), Martian volatiles-Their degassing history and geochemical fate, Icarus, 28, 179-202.

Farmer, C. B., and P. E. Doms (1979), Global and seasonal variation of water vapor on Mars and the implications for permafrost, J. Geophys. Res., 84, 2881-2888.

Feldman, W. C., et al. (2002), Global distribution of neutrons from Mars: Results from Mars Odyssey, Science, 297, 75-78.

Feldman, W., et al. (2004), Global distribution of near-surface hydrogen on Mars, J. Geophys. Res., 109, E09006, doi:10.1029/2003JE002160.

Golombek, M. P., et al. (2003), Selection of the Mars Exploration Rover landing sites, J. Geophys. Res., 108(E12), 8072, doi:10.1029/ 2003JE002074.

Haberle, R. M., and B. M. Jakosky (1991), Atmospheric effects on the remote determination of thermal inertia on Mars, Icarus, 90, 187-204.

Hauber, E., et al. (2005), Discovery of a flank caldera and very young glacial activity at Hecates Tholus, Mars, Nature, 434, 356

Head, J., J. Mustard, M. Kreslavsky, R. Milliken, and D. Marchant (2003), Recent ice ages on Mars, Nature, 426, 797-802.

James, P., B. Cantor, and S. Davis (2001), Mars Orbiter Camera observations of the Martian south polar cap in 1999-2000, J. Geophys. Res., 106, 23,635-23,652.

Kieffer, H. H., T. Z. Martin, A. R. Peterfreund, B. M. Jakosky, E. M. Miner, and F. D. Palluconi (1977), Thermal and albedo mapping of Mars during the Viking primary mission, J. Geophys. Res., 82, 4249-4291.

Kreslavsky, M. A., and J. W. Head (2003), North-south topographic slope asymmetry on Mars: Evidence for insolation-related erosion at high obliquity, Geophys. Res. Lett., 30(15), 1815, doi:10.1029/2003GL017795.

Kreslavsky, M., and J. W. Head (2005), Mars at very low obliquity: Atmospheric collapse and the fate of volatiles, Geophys. Res. Lett., 32, L12202, doi:10.1029/2005GL022645.

Leighton, R. R., and B. C. Murray (1966), Behavior of carbon dioxide and other volatiles on Mars, Science, 153, 136-144.

Mellon, M., and B. Jakosky (1993), Geographic variations in the thermal and diffusive stability of ground ice on Mars, J. Geophys. Res., 98, 3345-3364.

Mellon, M., and B. Jakosky (1995), The distribution and behavior of Martian ground ice during past and present epochs, J. Geophys. Res., 100, 11,781-11,799.

Mellon, M., B. Jakosky, and S. Postawko (1997), The persistence of equatorial ground ice on Mars, J. Geophys. Res., 102, 19,357-19,369.

Mellon, M. T., W. C. Feldman, and T. H. Prettyman (2004), The presence and stability of ground ice in the southern hemisphere of Mars, Icarus, 169, 324-340.

Mikovich, S., and J. Head (2003), Olympus Mons fan shaped deposit morphology: Evidence for debris glaciers paper presented at 6th International Mars Conference, Calif. Inst. of Technol., Pasadena.

Mitrofanov, I., et al. (2002), Maps of subsurface hydrogen from the high energy neutron detector, Mars Odyssey, Science, 297, 78-81.

Paige, D. A. (1992), The thermal stability of near-surface ground ice on Mars, Nature, 356, 43-45.

Putzig, N. E., M. T. Mellon, K. A. Kretke, and R. E. Arvidson (2005), Global thermal inertia and surface properties of Mars from the MGS mapping mission, Icarus, 173, 325-341. 
Rifkin, M. K., and J. F. Mustard (2001), Global distribution of unique surface processes imaged by the Mars Orbital Camera, Lunar Planet. Sci., XXXII, abstract 1698 .

Schorghofer, N., and O. Aharonson (2005), Stability and exchange of subsurface ice on Mars, J. Geophys. Res., 110, E05003, doi:10.1029/ 2004JE002350.

Schorghofer, N., and K. S. Edgett (2006), Seasonal surface frost at low latitudes on Mars, Icarus, 180, 321-334.

Smith, M. D. (2002), The annual cycle of water vapor on Mars as observed by the Thermal Emission Spectrometer, J. Geophys. Res., 107(E11), 5115, doi:10.1029/2001JE001522.
Zent, A. P., F. P. Fanale, and J. R. S. S. E. Postawko (1986), Distribution and state of $\mathrm{H}_{2} \mathrm{O}$ in the high-latitude shallow subsurface of Mars, Icarus, $67,19-36$

O. Aharonson, Division of Geological and Planetary Sciences, California Institute of Technology, MC 150-21, Pasadena, CA 91125, USA. (oa@gps.caltech.edu)

N. Schorghofer, Institute for Astronomy, University of Hawaii at Manoa, 2680 Woodlawn Drive, Honolulu, HI 96822, USA. (norb1@ifa.hawaii.edu) 